strains have been isolated in Japan. Horiuchi and colleagues reported in a recent issue of Antimicrobial Agents and Chemotherapy that seven strains of Shigella had sparfloxacin and ciprofloxacin MICs of approximately $0.4 \mu \mathrm{g} / \mathrm{mL}$, with slightly higher resistance to ofloxacin and with high-level resistance to naladixic acid $(>100 \mu \mathrm{g} / \mathrm{mL})$.

Although the MICs of resistant strains were within the breakpoint published by the NCCLS (for example, $\leqslant 2 \mu \mathrm{g} / \mathrm{mL}$ ofloxacin), the authors reported that three patients infected by relatively sparfloxacinresistant strains did not have pathogens eradicated after sparfloxacin therapy. Susceptible strains have extremely low MICs, in the range of 0.001 to 0.01 $\mu \mathrm{g} / \mathrm{mL}$. The Shigella sonnei strains reported here were 16 to 32 times less susceptible to fluoroquinolones.

Nearly all strains that cause shigellosis in the United States are $S$ sonnei and are uniformly susceptible to fluoroquinolones. Most laboratories report breakpoint susceptibilities of $S$ sonnei strains and do not detect relatively resistant strains.

Because agents like fluoroquinolones have very low MICs against enteric bacteria, it is unclear whether MICs a log or two higher indicate resistant strains. This report by Horiuchi provides some clinical evidence that certain strains treated with sparfloxacin are not eradicated.

FROM: Horiuchi S, et al. Antimicrob Agents Chemother 1993;37:2486-2489.

\section{AHCPR Releases Clinical Practice Guidelines for Managing HIV Infection}

The U.S. Department of Health and Human Services announced the release of new clinical practice guidelines to assist family physicians and other primary care practitioners in diagnosing and treating individuals with HIV infection. These guidelines were developed by a 19-member, private sector panel of medical experts and persons living with HIV for the Agency for Health Care Policy and Research (AHCPR), a part of HHS's Public Health Service. For additional information, contact AHCPR Bob Isquith at (301) 594-1364, extension 173.

\section{Paul Named to Replace Fauci as Head of AIDS Research Office}

Dr. William E. Paul, a scientist at the National Institute of Allergy and Infectious Disease (NIAID) in Bethesda, MD, will take over the newly restructured
Office of AIDS Research from Dr. Anthony S. Fauci, an AIDS researcher. Dr. Paul, a highly regarded immunologist, will oversee the entire federal budget for AIDS studies, which this year comes to \$1.3 billion. He will be responsible for shaping the direction of the research and determining how best to distribute the funds among the 21 institutes at the National Institutes of Health (NIH).

Dr. Paul, who has been at the NIH for more than 25 years, has not been involved directly in AIDS research. But, because his work deals with the immune system, it could end up being relevant to the disease. He has published more than 400 scientific papers, the most celebrated of which were those describing his laboratory's discovery of interleukin-4, one of the essential signaling molecules of the immune system.

Dr. Fauci had run the Office of AIDS Research in a smaller and less formalized version since 1988, while carrying out other duties as an administrator and scientist, but when Congress restructured the office last spring, it demanded that a full-time director be chosen. Scientists and advocates of AIDS research have expressed enthusiasm for the selection of Dr. Paul.

FROM: New York Times February 17, 1994.

\section{FDA Considers New Rules for Reuse of Single-Use Hemodialyzers}

The Food and Drug Administration (FDA) has proposed guidelines that would require manufacturers of single-use dialyzer products to test, label, and obtain new FDA approvals for multiple use of their products. Human test subjects would have to be stratified by age, race, sex, length of time receiving dialysis treatment, dialyzer type, and clinical cause of the end-stage renal disease (ESRD).

Although many manufacturers label and distribute their dialyzers for single use, the FDA believes that reprocessing for reuse has become so widespread that manufacturers should be required to qualify single-use dialyzers by the same standards that apply to dialyzers for multiple uses. The draft guidance document would go further than current requirements, however, in requiring manufacturers to monitor ESRD facilities' reprocessing for reuse. FDA data show that $70 \%$ of ESRD facilities have some form of reuse and that $80 \%$ of U.S. dialyzers are reused.

The Health Industry Manufacturers Association (HIMA) has challenged the FDA's authority to compel compliance with the proposed guidelines for hemodialyzer approval, labeling, and reprocessing for reuse, and may take legal action to block the guide- 
lines. HIMA has objected to the proposal on the basis that manufacturers' oversight of physicians' use of their products is inappropriate; because the proposed requirements would require lengthy and expensive testing of single-use products that ESRD facilities are reprocessing; because the FDA's current workload delays approvals for 400 days, and the addition of new applications for already-approved dialyzers would significantly lengthen the delay for all devices; and because the FDA may not have the authority to force compliance with the guidelines.

FROM: AAMI News February 1994.

\section{Penicillin Tolerated by Majority of Patients with History of Allergy}

Dr. Gadde and colleagues in Baltimore found that 97\% of study patients with a history of penicillin allergy and negative skin tests were able to tolerate treatment with penicillin. In a 5-year surveillance program for penicillin allergies in an STD clinic, 8.3\% of the 9,313 subjects enrolled reported a previous adverse reaction to penicillin. These reactions varied from anaphylaxis, urticaria, or exanthematous rashes to uncertain histories. All subjects with histories of penicillin allergy were skin-tested, and approximately $50 \%$ of the subjects without this history also were skin-tested. Penicillin skin-testing was performed in duplicate with benzyl penicilloyl polylysine (PPL), which is the major determinant, and a minor determinant mixture (MDM). Subjects who were positive to the MDM also were skin-tested with its individual constituents. All subjects with negative skin tests in both groups were treated with penicillin.

Of the 776 history-positive subjects who were skin tested, $7.1 \%$ reacted to either PPL or MDM or both, and $2.7 \%$ had equivocal reactions. Of the 4,287 subjects without a history of penicillin allergy who were skin-tested, significantly fewer $(1.7 \%)$ had positive reactions and $0.3 \%$ had equivocal reactions. Of all the subjects with positive skin tests, $75 \%$ reacted to PPL alone, $10.2 \%$ to MDM alone, and $14.8 \%$ to both PPL and MDM. Of the subjects with equivocal reactions, $82 \%$ were retested later, and all were found to have negative skin tests.

The reactions were classified according to the type of prior reaction. Positive skin tests occurred in $17.3 \%$ of patients with a history of anaphylaxis, $12.4 \%$ of patients with a history of urticaria, $4 \%$ of patients with a history of exanthematous rash, and $1.7 \%$ of patients with an uncertain history.

This study confirms the need for penicillin skin testing to verify or exclude the diagnosis of penicillin allergy

FROM: Gadde J, et al. JAMA 1993;270:2456-2463. 